\title{
Eplerenone, amlodipine and experimental hypertension: one plus one equals three
}

\author{
John W Funder \\ Hypertension Research (2011) 34, 795-796; doi:10.1038/hr.2011.60; published online 26 May 2011
}

$\mathrm{T}$ here are at least three reasons for writing an Editorial Commentary on the paper by Nakamura et al., ${ }^{1}$ published in this issue of the Journal 'Eplerenone potentiates protective effects of amlodipine against cardiovascular injury in salt-sensitive hypertensive rats'.

The first is because it is a model of what a very good paper should be. It is a comprehensive account of the differential (and additive) effects of two agents in protecting the heart and blood vessels, exploring a range of outcomes and possible mediating mechanisms. In addition to being comprehensive (in the allowable seven figures there are a total of over 20 panels, all informative, none inviting a reviewer to suggest they be dropped) it is both logical and linear. The logic is unassailable, and the linearity in the narrative super smooth: the data are presented in such a way that the reader both expects and is looking forward to the next set of results and figures. Finally, the discussion is relatively brief, focused and modest; again, the narrative is logical and linear, and a pleasure to read. The authors are to be congratulated on what they have performed, and how they have reported their findings: for students, fellows (and their supervisors) the manuscript should serve as a model of how to write a scientific report.

The second reason for an Editorial Commentary is that the findings reported reinforce and extend our appreciation of the role of MR activation in salt-induced experimental hypertension. MR antagonists (spironolactone and eplerenone) have been historically classified as potassium-sparing diuretics, and their effects attributed (often almost subcon-

Professor JW Funder is at the Prince Henry's Institute, PO Box 5152, Clayton, Victoria 3168, Australia.

E-mail: john.funder@princehenrys.org sciously) to natriuresis and protection by elevated plasma $\left[\mathrm{K}^{+}\right]$. Fortunately this historical aberration has been successfully challenged by both experimental and clinical studies, largely using eplerenone. In the paper under review, amlodipine lowered blood pressure (BP) (and LV hypertrophy), improved vascular endothelial function by a range of measures (eNOS phosphorylation, Akt phosphorylation, plasma NOx and vascular superoxide), but was without effect on others (vascular NADPH oxidase, gp91 ${ }^{\text {phox }}$ AT1 receptor levels). In contrast, eplerenone at the dose used did not lower BP, but did reduce levels of vascular NADPH oxidase activity, gp91 phox and vascular AT1 receptors. Combination therapy with the two agents, perhaps predictably (and comfortingly) showed additive effects.

The effects in the heart were similar, but not identical to those in the vascular wall. Cardiac functional parameters were improved by amlodipine but not eplerenone: both reduced cardiac macrophage infiltration, MCP-1 expression, cardiac interstitial fibrosis, TGF $\beta 1$ mRNA expression and cardiac superoxide. Eplerenone, but not amlodipine, reduced cardiac gp91 ${ }^{\text {phox }}$ and NADPH oxidase activity, as well as AT1 receptor levels, as it had done in the vessel wall.

Importantly, these effects were seen with no differences between treatment groups in plasma electrolytes, osmolarity or aldosterone levels, nor in urinary volume, osmolarity or sodium excretion: so much for the effects of MR blockade reflecting its function as 'a potassium-sparing diuretic'. What is also important is that the high-salt intake lowered plasma aldosterone levels, and yet MR blockade was clearly effective. The authors postulate that this may reflect Rac1 activation, known in turn to activate MR in the absence of aldosterone, although MR activa- tion is blocked by MR antagonists; they also acknowledge the effects of endogenous glucocorticoids in activating MR in the context of tissue damage. The bottom line is that in the absence of BP lowering and in conditions where aldosterone levels are suppressed (but arguably not to low enough levels), MR blockade is clearly vasculo- and cardioprotective, by mechanisms that overlap with but in large part are clearly distinct from $\mathrm{Ca}^{++}$channel blockade and the consequent $\mathrm{BP}$ reduction.

The third reason for an Editorial Commentary is to briefly put forward what these studies may mean for clinical medicine. Eplerenone in man is much more BP active than in the rat, as shown by comparability studies versus ACE inhibition (enalapril) and $\mathrm{Ca}^{++}$channel blockade (amlodipine). There are two other findings of relevance: first, that it is more vasoprotective, at equivalent BP-lowering doses, than enalapril or amlodipine; second, in so-called resistant hypertension, MR blockade is particularly effective, with BP reductions of up to $30 \mathrm{~mm} \mathrm{Hg}$.

Where this is relevant is in the context of primary aldosteronism, now recognized to constitute $\sim 10 \%$ of hypertension worldwide. Even in developed countries with excellent publicly supported health systems, a miniscule percentage (certainly below 1\%) of this $10 \%$ is screened for primary aldosteronism each year. In a lightly populated country (22 million people) like Australia, there are possibly between a quarter and half a million subjects with autonomous aldosterone secretion; in more populated countries there are millions.

We have focused on screening, case detection, lateralization and management-of the tip of this iceberg. There is incontrovertible evidence that primary aldosteronism has 
far more serious implications in terms of cardiovascular damage than is seen in age-, sex- and BP-matched essential hypertensives. There is also very good evidence that MR blockade is particularly vasoprotective experimentally (as shown by Nakamura et al.) and clinically, in essential hypertension with normal or even low plasma aldosterone levels.

Put all this together-the tip of the iceberg, the high levels of undiagnosed primary aldosteronism, its high levels of cardiovascular sequelae and the vasculo-protective effects of eplerenone in essential hypertension, we have the recipe for a paradigm shift. MR blockade should be part of first-line treatment of all patients with elevated blood pressure. Modest doses of eplerenone (25-50 mg pert day) will benefit patients with essential hypertension, without any problems of hyperkalemia unless renal function is severely compromised. For the $10 \%$ of such patients with occult primary aldosteronism, such treatment will be game changing. Primary aldosteronism is now a major, if under-recognized public health problem: we have the means to mitigate its effects, but not to diagnose it on a population basis. The message is clear.

\section{CONFLICT OF INTEREST}

The author declares no conflict of interest.

1 Nakamura T, Fukuda M, Kataoka K, Nako H, Tokutomi Y, Dong Y-F, Yamamoto E, Yasuda O, Ogawa H, KimMitsuyama S. Eplerenone potentiates protective effects of amlodipine against cardiovascular injury in saltsensitive hypertensive rats. Hypertens Res 2011; 34: 817-824. 